\title{
Involvement of Macrophage Migration Inhibitory Factor (MIF) in Experimental Uric Acid Nephropathy
}

\author{
Yoon-Goo Kim, ${ }^{1,2}$ Xiao-Ru Huang, ${ }^{3}$ Shin-ichi Suga, ${ }^{1}$ Marilda Mazzali, ${ }^{1}$ \\ Dongjiang Tang, ${ }^{3}$ Christine Metz, ${ }^{4}$ Richard Bucala, ${ }^{4}$ Salah Kivlighn, ${ }^{5}$ \\ Richard J. Johnson' ${ }^{1}$ and Hui Y. $\operatorname{Lan}^{3}$ \\ ${ }^{1}$ Division of Nephrology, University of Washington Medical Center, Seattle, \\ Washington, U.S.A. \\ ${ }^{2}$ Samsung Medical Center, Sungkyunkwan University, Seoul, Korea \\ ${ }^{3}$ Department of Medicine, University of Hong Kong, Queen Mary Hospital, \\ Hong Kong \\ ${ }^{4}$ The Picower Institute for Medical Research, Manhasset, New York, U.S.A. \\ ${ }^{5}$ Merck and Company, Inc., West Point, Pennsylvania, U.S.A. \\ Communicated by R. Bucala. Accepted June 20, 2000.
}

\begin{abstract}
Background: Deposition of uric acid in the kidney can lead to progressive tubulointerstitial injury with granuloma formation. We hypothesized that uric acid crystal deposition may induce granuloma formation by stimulating local expression of macrophage migration inhibitory factor (MIF), which is a known mediator of delayed type hypersensitivity (DTH).

Materials and Methods: A model of acute uric acid nephropathy was induced in rats by the administration of oxonic acid (an inhibitor of uricase), together with uric acid supplements. MIF expression and local cellular response were examined by in situ hybridization and immunohistochemistry.

Results: Kidney tissue examined at $\mathbf{3 5}$ days posttreatment showed widespread tubulointerstitial damage with intratubular uric acid crystal deposition and granuloma formation. Tubules within the areas of granuloma showed a six-fold increase in MIF mRNA, compared with uninvolved areas by in situ hybridization. Moreover, the areas of
\end{abstract}

increased MIF mRNA expression correlated with sites of dense accumulation of macrophages and $T$ cells, and these cells were activated when assessed by the expression of interleukin-2R (IL-2R) and (MHC) class II. Interestingly, cytoplasmic staining for MIF protein in the uric acid (UA) crystalassociated granulomatous lesions was reduced, indicating a rapid MIF secretion by damaged tubules and macrophages secondary to uric acid crystal stimulation. This was confirmed by the demonstration of a marked increase in urinary MIF protein by Western blot analysis. Control rats fed either a normal diet or only oxonic acid had no discernible evidence of renal disease by routine light microscopy and minimal tubular expression of MIF mRNA and protein.

Conclusions: These data suggest that intrarenal granulomas in urate nephropathy may be the consequence of a crystal induced DTH reaction mediated by MIF.

\section{Introduction}

Patients with hyperuricemia are at risk for developing both acute and chronic renal disease.

Address correspondence and reprint requests to: Dr Hui Y. Lan, University Department of Medicine, Queen Mary Hospital, Pokfulam, Hong Kong. Phone: 852-2855-3459; Fax: 852-2855-1143; E-mail: hylan@hkucc.hku.hk
An acute urate nephropathy has been described in the setting where massive urate overproduction leads to marked hyperuricosuria with intratubular crystal formation and acute renal failure. This syndrome classically is observed in patients with myeloproliferative disorders, particularly after chemotherapy or radiation has induced rapid cell lysis. The 
pathogenesis is thought to be due to primarily tubular obstruction by uric acid crystals and is often considered reversible with appropriate treatment $(1,2)$.

Patients with longstanding hyperuricemia also may develop modest renal insufficiency, in which the renal lesion is that of chronic tubulointerstitial disease, often with urate crystal deposition in the outer medulla. These crystals are usually localized within collecting ducts and/or in the interstitium, and are often surrounded by a granulomatous reaction containing leukocytes and giant cells (3-5). Although it remains controversial as to whether the generalized tubulointerstitial fibrosis is a consequence of crystalinduced renal damage, as opposed to renal injury from the frequently coexistent hypertension, the focal crystal deposition is thought to be responsible for the local granuloma formation (6-8).

The observation that uric acid crystal deposition can be associated with granuloma formation suggests to us that the uric acid crystals may act to stimulate a delayed-type hypersensitivity (DTH)-like reaction. In this regard, one of the most important cytokines driving the DTH reaction is macrophage migration inhibitory factor (MIF). MIF originally was described as a product of activated $\mathrm{T}$ cells that inhibited the random migration of guinea pig peritoneal macrophages in vitro and promoted macrophage accumulation in the DTH reaction $(9,10)$. Recently, the functional role of MIF in the immune and inflammatory responses was determined using neutralizing antibodies. In vivo blocking studies have shown that MIF plays a pivotal role in the tuberculin-induced skin DTH reaction (11), experimental models of arthritis (12), endotoxic shock $(13,14)$, and can act as a counter-regulator of glucocorticoid action (15). Furthermore, MIF now has been shown to be produced by intrinsic kidney cells, including renal tubular cells and mesangial cells $(16,17)$, and upregulation of MIF in the kidney has been demonstrated to play an important role in both experimental and human immunologic kidney disease, including renal allograft rejection (18-25).

Therefore, the aim of current study was to examine whether the sites of uric acid crystal deposition might induce local MIF expression as a mechanism for inducing a local granulomatous reaction.

\section{Materials and Methods}

\section{Experimental Disease Model}

Male Sprague-Dawley rats weighing 225 to $250 \mathrm{~g}$ (Simonsen Laboratory, Gilroy, CA) were used in this study. Experimental uric acid nephropathy was induced by feeding $2 \%$ oxonic acid (OA) and $1.5 \%$ uric acid supplements for 35 days (UA group). Control rats were fed only $2 \%$ OA supplements (OA group) or a normal commercial diet (NC group) for 35 days. The administration of oxonic acid was necessary as rats have uricase, a hepatic enzyme that degrades uric acid to a more soluble allantoin (26). Rats were allowed free access to food and tap water. Six rats were studied per group.

Blood and $24 \mathrm{hr}$ urine collections were taken at days $0,7,14$, and 35 . Rats were sacrificed at day 35 . The sera and urine were stored at $-20^{\circ} \mathrm{C}$ until measured.

\section{Measurement of Uric Acid and Blood Urea Nitrogen (BUN)}

Uric acid in serum and urine was measured by a modified phosphotungstate method (27). Blood urea nitrogen (BUN) was determined colorimetrically using a commercial kit for the measurement of urea nitrogen (Sigma Diagnostics, St. Louis, MO).

\section{Renal Histology}

Kidneys obtained at the time of sacrifice were divided and fixed in methyl Carnoy's solution, $10 \%$ buffered formalin, and absolute ethanol. Methyl Carnoy's solution-fixed tissues were paraffin-embedded and sectioned $(4 \mu \mathrm{m})$ and stained with the periodic acid/Schiff (PAS) reagent with hematoxylin. To preserve uric acid crystals, absolute ethanol-fixed tissues were placed into a solution composed of equal parts of xylene and paraffin for $2 \mathrm{hr}$ at $58^{\circ} \mathrm{C}$. The tissues were then transferred into paraffin for $1 \mathrm{hr}$ at $58^{\circ} \mathrm{C}$ and, subsequently, embedded. Sections cut at $8 \mu \mathrm{m}$ thick were stained by the DeGalantha method to identify uric acid crystals. Before the tissues were stained, the paraffin was removed by the brief placement of the slide in xylene and then washed in absolute ethanol (28).

A point counting technique was used to quantify tubulointerstitial damage (tubular atrophy, leukocyte infiltration, and fibrosis) in at least 50 high-power fields on PAS-stained sections (29). 


\section{Antibodies and Probe}

The following mouse monoclonal antibodies (mAbs) were used: IIID9, mouse anti-MIF mAb [immunoglobulin Gl (IgGl) subclass] raised to purified recombinant mouse MIF and cross-reactive with human MIF (16-25); EDl, mouse anti-rat CD68 labeling most monocytes and macrophages (30); R73, recognized a nonpolymorphic epitope of the $\alpha \beta \mathrm{T}$ cell receptor (TCR) (31); NDS 61, anti-rat CD25, p55 chain of the rat IL-2R (32); OX-6, anti-rat major histocompatibility complex (MHC) class II (33); W3/13, recognized leukocytes, including T cells and polymorphonuclear neutrophils (PMNs)(34). Peroxidase and alkaline phosphatase-conjugated goat anti-mouse IgG, mouse peroxidase anti-peroxidase complexes (PAP), and mouse alkaline phosphatase anti-alkaline phosphatase complexes (APAAP) were purchased from Dakopatts (Glostrup, Denmark).

A 420 base pair fragment of mouse MIF cDNA cloned into pBluescript (Strategene, La Jolla, CA) was used to prepare digoxigenin (DIG)-labeled anti-sense and sense cRNA probes for in situ hybridization according to the manufacturer's protocol (BoehringerMannheim Biochemica, Mannheim, Germany).

\section{Immunohistochemistry}

One- and two-color immunohistochemical staining using mAbs to MIF (III.D.9), macrophage (ED1), TCR (R73), IL-2R (CD25), MHC class II (OX-6), and T cells/neutrophils (W3/13) was performed on $4 \mu \mathrm{m}$ paraffin sections of formalinfixed tissue using a previously described microwave-based method (16-25,35).

Briefly, sections were treated two times for $5 \mathrm{~min}$ of microwave oven heating in $0.01 \mathrm{M}$ sodium citrate, $\mathrm{pH} 6.0$, at $2450 \mathrm{MHz}$ and 800 watts. This procedure facilitated retrieval of cytoplasmic antigens. Sections then were preincubated with $10 \%$ fetal calf serum (FCS) and $10 \%$ normal goat serum in phosphate-buffered saline (PBS) for $20 \mathrm{~min}$, drained, and labeled with mouse mAbs for $60 \mathrm{~min}$, washed (three times) in PBS, and endogenous peroxidase inactivated by incubation in $0.3 \% \mathrm{H}_{2} \mathrm{O}_{2}$ in methanol. Sections then were washed in PBS, incubated with peroxidase-conjugated goat anti-mouse IgG for $60 \mathrm{~min}$, washed in PBS, incubated with mouse peroxidase antiperoxidase complexes, and developed with 3,3-diaminobenzidine to produce a brown color.
For double immunostaining of EDI and MIF, sections were microwave heated a second time after the first round of EDl antibody staining, which denatured bound IgG molecules on the sections and prevented antibody cross reaction (34). Sections then were preincubated as above and labeled with the antiMIF mAb overnight at $4^{\circ} \mathrm{C}$. After washing in PBS, sections were incubated sequentially with alkaline phosphatase-conjugated goat anti-mouse IgG and mouse alkaline phosphatase anti-alkaline phosphatase complexes, and then developed with Fast Blue BB Salt (Ajax Chemicals, Melbourne, Australia). Sections were counterstained with periodic acid-Schiff (PAS) reagent and mounted in an aqueous medium. An isotype-matched mouse anti-human CD45R mAb(73.5) that did not react with rat tissues was used as a negative control.

\section{In situ Hybridization}

In situ hybridization for MIF mRNA was performed on $4 \mu \mathrm{m}$ paraffin sections of formalinfixed tissue using a microwave-based protocol (16-25,36). After dewaxing, sections were treated with a microwave oven two times for $5 \mathrm{~min}$, as outlined above, incubated with $0.2 \mathrm{M}$ $\mathrm{HCl}$ for $15 \mathrm{~min}$, followed by $1 \%$ Triton X-100 for $15 \mathrm{~min}$, and then digested for $20 \mathrm{~min}$ with $10 \mu \mathrm{g} / \mathrm{ml}$ proteinase $\mathrm{K}$ at $37^{\circ} \mathrm{C}$ (BoehringerMannheim Biochemica). Sections then were washed two times in (SSC), pre-hybridized, and hybridized with $0.5 \mathrm{ng} / \mu \mathrm{l}$ digoxigeninlabeled sense or anti-sense cRNA probe overnight at $42^{\circ} \mathrm{C}$ in a hybridization buffer containing $50 \%$ deionized formamide, $4 \times$ SSC, $1 \mathrm{mg} / \mathrm{ml}$ salmon sperm DNA (Sigma), and $1 \mathrm{mg} / \mathrm{ml}$ yeast tRNA. Sections were washed finally in $0.1 \times \mathrm{SSC}$ at $42^{\circ} \mathrm{C}$ and the hybridized probe detected using alkaline phosphataseconjugated sheep anti-digoxigenin $\mathrm{F}(\mathrm{ab})$ fragments and color development with nitro blue tetrazolium/X-phosphate. In addition, some sections were treated with microwave oven heating again and then stained with the EDI mAb using the three-layer PAP method as described above. Sections were counterstained with PAS and mounted in an aqueous medium.

Controls employed a sense MIF cRNA probe labeled to the same specific activity as the anti-sense probe, or omitted the probe completely. No staining was seen in either normal or diseased kidney using the sense probe or with no probe at all. 


\section{Western Blot Analysis}

To measure MIF secretion by damaged tubular epithelial cells and activated macrophages and $\mathrm{T}$ cells, urinary samples $(5 \mathrm{ml})$ were mixed with SDS-PAGE sample buffer, boiled for $5 \mathrm{~min}$, electrophoresed on a $10 \%$ SDS polyacrylamide gel, and electroblotted onto Hybond-ECL nitrocellulose membrane (Amersham International, Buckinghamshire, U.K.). The membrane was blocked in PBS containing 5\% skimmed milk powder, $1 \%$ FCS, $0.02 \%$ Tween 20 , and then incubated for $1 \mathrm{hr}$ with rabbit anti-murine MIF polyclonal antibody (1:1500) diluted in the above buffer. After washing, the membrane was incubated with a 1:20,000 dilution of peroxidaseconjugated swine anti-rabbit IgG in PBS containing $1 \%$ normal swine serum and $1 \%$ FCS. The blot was then developed using the ECL detection kit (Amersham) to produce a chemiluminescence signal that was captured upon X-ray film.

\section{Quantitation of Tissue Staining}

A semi-quantitative method was used to quantify glomerular and tubulointerstitial cells stained by mAbs or the cRNA probe as described previously (16-25).

To assess tubulointerstitial and glomerular staining, the number of labeled cells was counted in the randomly selected areas of the tissue under high power fields (40X) by means of a $0.02-\mathrm{mm}^{2}$ graticule fitted in the eyepiece of the microscope, and expressed as cells per $\mathrm{mm}^{2}$ or cells per glomerular crosssection. Tubular MIF mRNA expression was scored from 500-1000 tubules and expressed as percentage of positive tubules. In addition, a point counting method was used to quantitate renal MIF expression, macrophage $\left(\mathrm{ED} \mathrm{l}^{+}\right)$and $\mathrm{T}$ cell $\left(\mathrm{R}^{+} 3^{+}\right)$infiltration and activation (IL-2R and MHC class II expression) in UA-induced granulomatous lesions. Briefly, at least 10-20 granulomatous lesions were examined for each animal in mAb-labeled tissue sections of the kidney. All positive cells within granulomas and non-granulomatous tissue were scored under high power fields $(40 \mathrm{X})$ by means of a $0.02-\mathrm{mm}^{2}$ graticule fitted in the eyepiece of the microscope and expressed as cells per $\mathrm{mm}^{2}$. For each tissue, the same area was examined in serial sections labeled with different mAbs. No adjustment of the interstitial cell count was made for tubules or the luminal space. All counting was performed on blinded slides. Most tubules showed no or very weak detection of MIF protein by the anti-MIF mAb within the lesions, due to a rapid release of tubular MIF upon uric acid crystal stimulation. Therefore, no data for MIF protein staining were shown in this study.

\section{Statistical Analysis}

One-way analysis of variance from the Complete Statistical Systems program (CSS; Statsoft, Tulsa, OK) was used to analyze differences in MIF expression, macrophage, and $\mathrm{T}$ cell accumulation and activation. The differences of serum and urinary uric acid levels and BUN between the groups were compared with unpaired Student's $t$-tests or ANOVA with the Fisher's protected least significant difference test for multiple comparison. Correlation between two variables was evaluated by linear regression analysis.

\section{Results}

\section{Serum Uric Acid and Urinary Uric Acid Excretion}

The mean serum uric acid level and $24 \mathrm{hr}$ urinary uric acid excretion of rats before feeding $\mathrm{OA}$ and/or UA supplements (day 0 ) was $1.33 \pm$ $0.05 \mathrm{mg} / \mathrm{dl}$ and $3.37 \pm 0.23 \mathrm{mg} /$ day. Rats fed oxonic acid (OA group) showed a mild increase in serum uric acid levels from days 7 to 35, compared with normal controls (Fig. 1A). In contrast, rats fed both oxonic acid and uric acid (UA group) showed much higher serum uric acid levels from days 7 to 35 . Peak uric acid levels were at day 14 (Fig. 1A).

Similarly, $24 \mathrm{hr}$ urinary uric acid excretion in OA group also was mildly increased, compared with NC group from days 7-35 (Fig. 1B). However, urinary uric acid excretion was increased markedly in the UA group from days 7-35, and this parameter peaked at day 14 (Fig. 1B).

\section{Renal Function and Histology}

Following the same pattern of a significant increase in serum and urinary uric acid levels from days 7-35, rats in the UA group exhibited a marked increase in serum BUN level at day 7 . The BUN levels remained increased until day 35, compared with the $\mathrm{OA}$ and the normal 

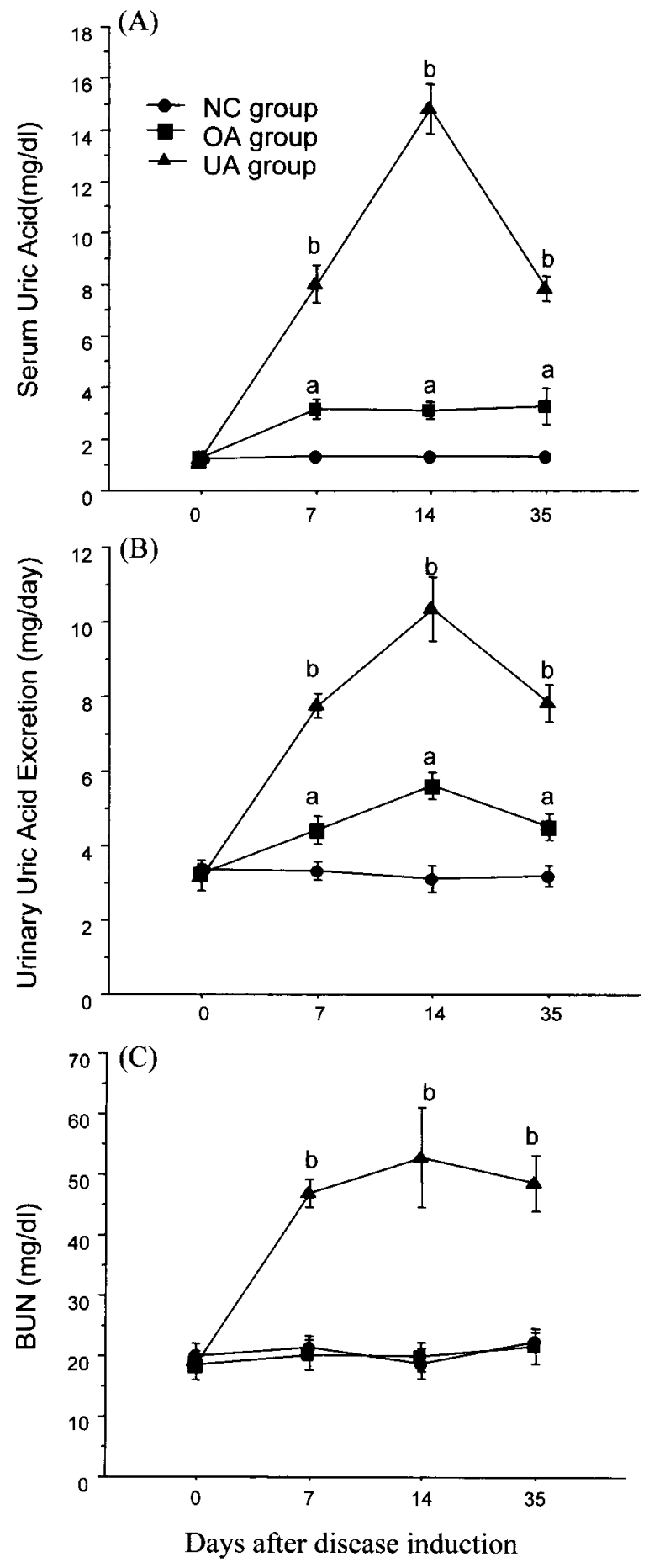

Fig. 1. Changes in uric acid levels and renal function. (A) Serum uric acid levels; (B) $24 \mathrm{hr}$ urinary uric acid excretion; and (C) Blood urea nitrogen (BUN) levels in rats fed normal commercial diet ( $\bullet$; NC group), oxonic acid ( $\square$; OA group) and oxonic acid plus uric acid supplement $(\Delta ;$ UA group). ${ }^{\mathrm{a}} p<0.05$ vs. NC group, ${ }^{\mathrm{b}} p<0.05$ vs. NC and OA groups. Data are mean \pm SEM. control groups (Fig. 1C). In contrast, the BUN in OA group remained normal (Fig. 1C).

OA animals showed a normal gross appearance, as well as histology by routine light microscopy (Fig. 2A). However, kidneys from UA group had an irregular cortical surface, with obvious pits or scars. The cut surface revealed yellow streaks in the pyramid in all six rats of the UA group. The tissue injury was confined to the tubulointerstitium and glomeruli appeared normal. In both cortex and medulla, there was severe interstitial mononuclear cell infiltration and fibrosis. Most tubules exhibited severe atrophy, dilatation, or hyperplasia. Within some tubules and interstitial areas, the accumulation of neutrophils also was apparent. Strikingly, some tubules contained varying amounts of inflammatory cells, cellular debris, and amorphous or acicular material. By the De Galantha stain, the precipitated amorphous material was confirmed to be collections of urate crystals (Fig. 2B). A typical granuloma formation was apparent, surrounding these amorphous deposits, in which the central accumulation of crystalloid material was surrounded by a rim of leukocytes, multinuclear giant cells, and fibroblasts (Fig. 2C).

Semi-quantitative analysis showed that there was minor tubulointerstitial lesions (tubular atrophy, and interstitial leukocyte infiltration and fibrosis) in the OA group (3.8 \pm $1.3 \%$ ), which was not significantly different, compared with normal controls $(1.8 \pm 1.4 \%$, $p=$ not significant). In contrast, the UA group demonstrated severe tubulointerstitial damage $(79.5 \pm 8.6 \%, p<0.001$ vs. NC and OA group).

\section{Constitutive MIF Expression in Normal Control and $O A$ Groups}

In situ hybridization and immunohistochemistry showed that MIF mRNA and protein were expressed constitutively in kidneys of the normal control and OA groups. A small number of glomerular visceral and parietal epithelial cells were positive for MIF MRNA, and exhibited weak MIF protein expression. Tubules $(>70 \%)$ also showed a weak expression of MIF protein; whereas, only a small portion of tubules $(10.3 \pm 2.7 \%)$ expressed MIF mRNA. In the OA group, both MIF mRNA $(13.2 \pm 4.4 \%)$ and protein expression were not different, compared with normal controls (Figs. 3A, 3B, 5A). 

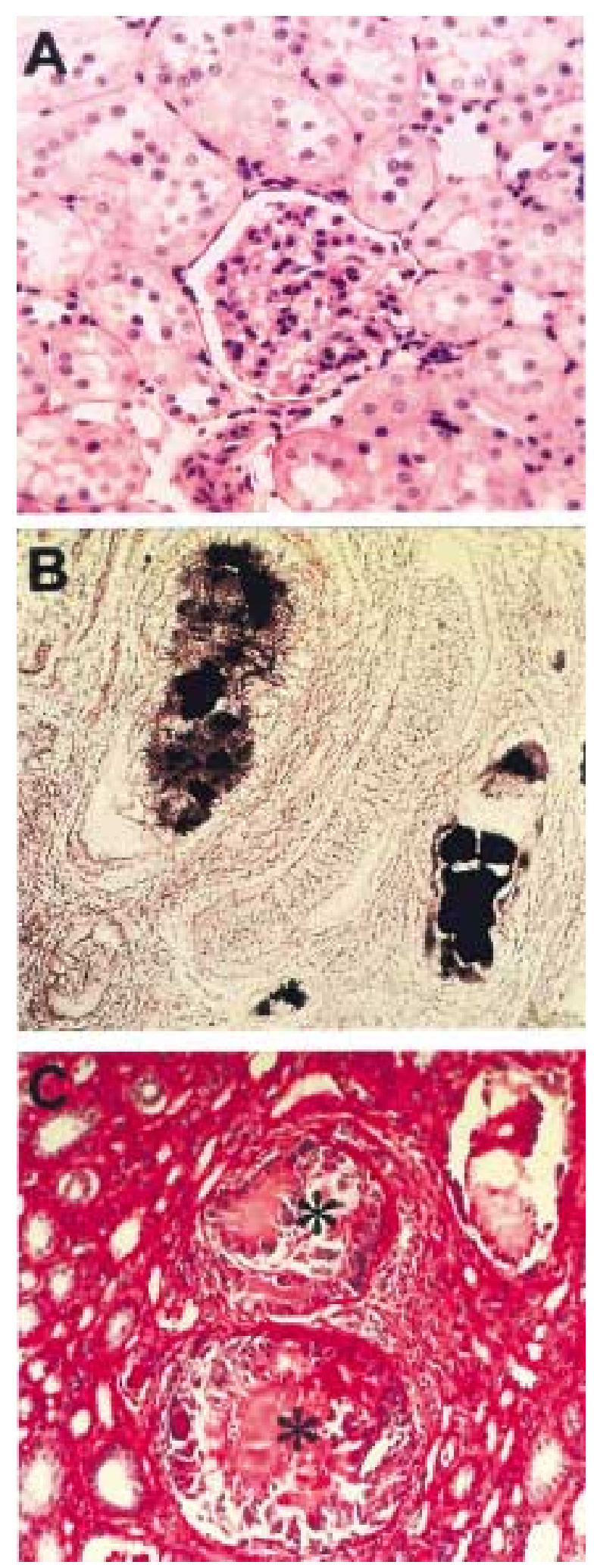

Fig. 2. Histological changes in urate nephropathy. (A) Normal kidney morphology from an oxonic acid (OA) rat (periodic acid/Schiff reagent; PAS, 200X). (B) Amorphous uric acid and acicular urate crystals within tubules in oxonic acid plus
Up-regulation of MIF Expression, Local Macrophage and $T$ cell Accumulation and Activation in the Tubulointerstitium in UA Nephropathy

In contrast to kidneys of normal control and OA groups (Figs. 3A, 3B and 5A), there was a marked (>6-fold) increase in MIF mRNA expression by renal tubules in the UA group (83 $\pm 8 \%$ of total tubules) and infiltrating macrophages, compared with normal and OA group (Figs. 3C-3F and 5A, $p<0.001$ ). The upregulation of renal MIF mRNA was largely limited to injured tubulointerstitial areas and was greatest within crystal-associated granulomas, as determined by in situ hybridization; whereas, there was no obvious change in constitutive MIF expression in those areas with mild or no evidence of injury (Fig. 3C). In contrast to the marked increase in MIF mRNA expression noted in the sites of severe tubulointerstitial damage, immunostaining for MIF protein was expressed weakly or negative within the lesions; whereas, constitutive MIF protein expression remained unaltered in those areas with mild or no evidence of tubulointerstitial injury (Fig. 3D). This suggested that MIF was synthesized rapidly, and released by the damaged tubular epithelial cells and activated macrophages and T cells. Consistent with this possibility, Western blot analysis showed a marked increase in urinary MIF protein levels in all UA animals; whereas, only low levels or undetectable urinary MIF were found in both normal and OA control animals (Fig. 4). There also was a 20-fold increase in $\mathrm{EDI}^{+}$macrophages and a 10-fold increase in $\mathrm{R}^{+} 3^{+} \mathrm{T}$ cells in the tubulointerstitium, compared with normal control and OA groups $(p<0.001)$ (Figs. 5B, 5C and 6A, 6B). Combined in situ hybridization and immunostaining showed that up-regulation of MIF mRNA expression in the tubulointerstitial lesion was associated spatially with the infiltration of $\mathrm{EDI}^{+}$macrophages (Figs. 3C, 3E). Similarly, most R73+ T cells also were present within the areas with severe tubulointerstitial damage (Figs. 6A, 6B). By linear regression analysis a strong correlation could be shown between MIF mRNA expression with macrophage $(r=0.975, p<0.001)$

uric acid (UA) group (De Galantha stain 400X). (B) Granuloma formation (*) with central accumulation of crystalloid material surrounded by leukocytes, multinucleated giant cells and fibrosis (PAS stain 200X). 

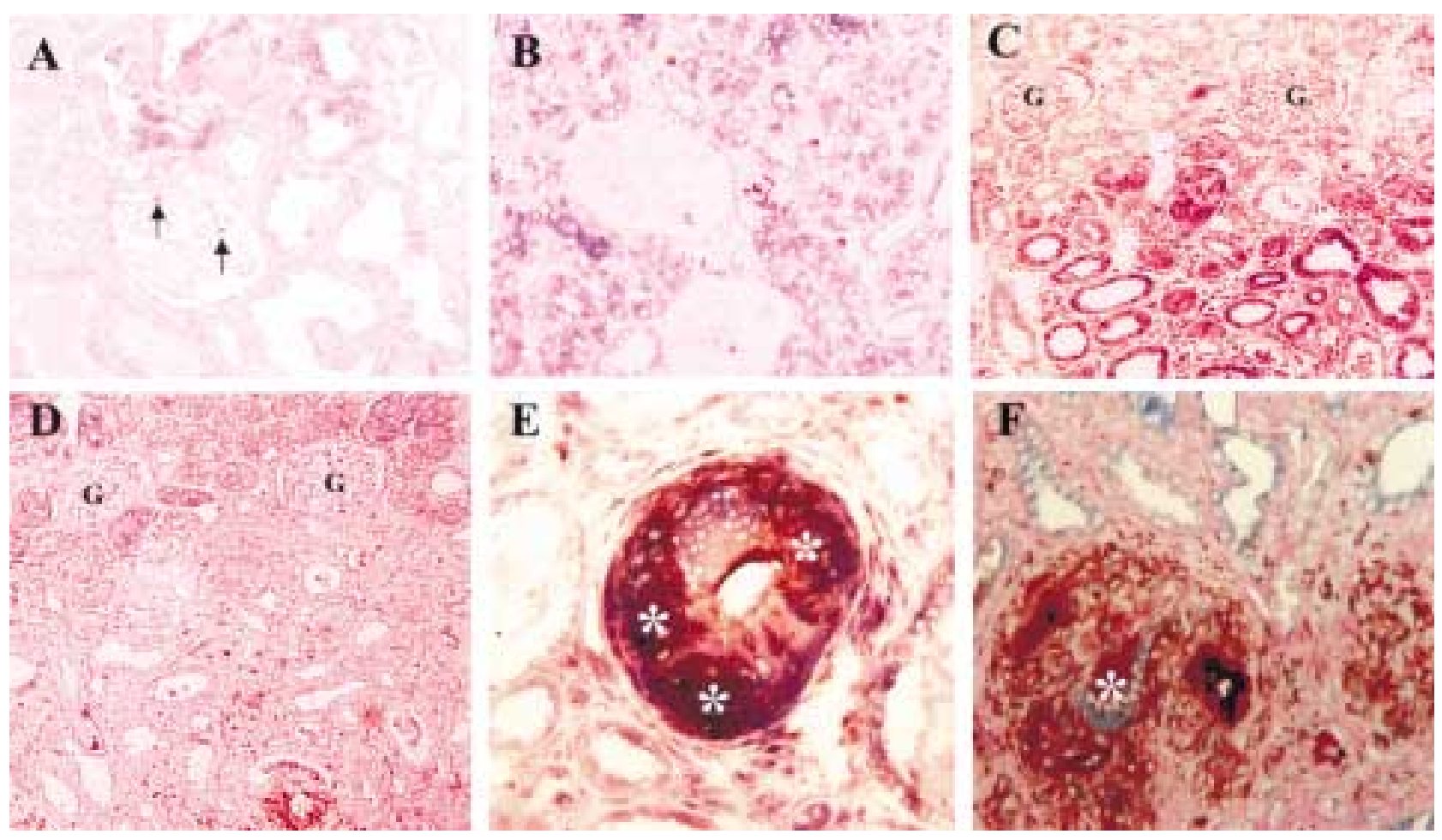

Fig. 3. Double in situ hybridization and immunohistochemistry detecting macrophage migration inhibitory factor (MIF) expression and macrophage accumulation. (A) Constitutive MIF mRNA expression (purple) by some glomerular and tubular epithelial cells in the kidney of oxonic acid (OA) group. Few ED ${ }^{+}$macrophages (brown, arrows) are found within the glomerulus and interstitium. (B) Constitutive weak MIF protein expression (blue) by most tubules with some $\mathrm{ED}^{+}$macrophages within glomeruli and occasional damaged tubules in the kidney of OA group. (C, D) Serial sections demonstrating differential up-regulation of MIF mRNA and protein expression in the early tubulointerstitial lesion in oxonic acid plus uric acid (UA) groups ( $\mathrm{G}=$ glomerulus). A marked up-regulation of MIF mRNA (C, purple) by tubular epithelial cells is limited to severe tubulointerstitial lesions with many EDI ${ }^{+}$macrophages (brown-purple) accumulation $(*)$. Note that many ED $1+$ macrophages also expressed MIF mRNA. In contrast, immunostaining of the serial sections demonstrated that there is very weak or undetectable MIF protein expression by the damaged tubules and macrophages (brown) within the lesions $(*)$; whereas, weak constitutive expression of MIF protein is unaltered in the areas of the kidney that are normal or minimally injured. (E) Strong MIF mRNA (purple) expression and macrophage accumulation (brown-purple) in a damaged tubules of UA groups, where some evidence for deposition of uric acid crystals was found within the lumen with marked tubular epithelial cell proliferation and MIF expression. Note that all macrophages $\left.{ }^{*}\right)$ within the damaged tubules also strongly express MIF mRNA (brown-purple). But, there is no MIF up-regulation and macrophage accumulation found in those non-inflamed tubules. (F) Macrophage-rich (brown) granuloma formation in UA group. Note that a multinucleated giant cells with strong MIF protein expression (blue) is found within the granuloma $(*)$. All sections are counterstained with periodic acid/Schiff reagent (PAS). Magnifications: 250X (A, B), 100X (C, D), 400X (E, F).

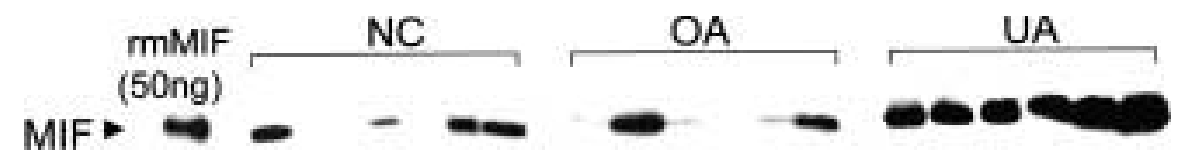

Fig. 4. Quantitation of urinary macrophage migration inhibitory factor (MIF) secretion. Western blot analysis demonstrated a marked increase in urinary MIF secretion in oxonic acid plus uric acid (UA) animals; whereas, low or undetectable urinary MIF levels were found in oxonic acid (OA) and normal control (NC) animals. Urine samples were collected at day 35, after induction of disease. Each bar represents one animal for groups of six rats. 

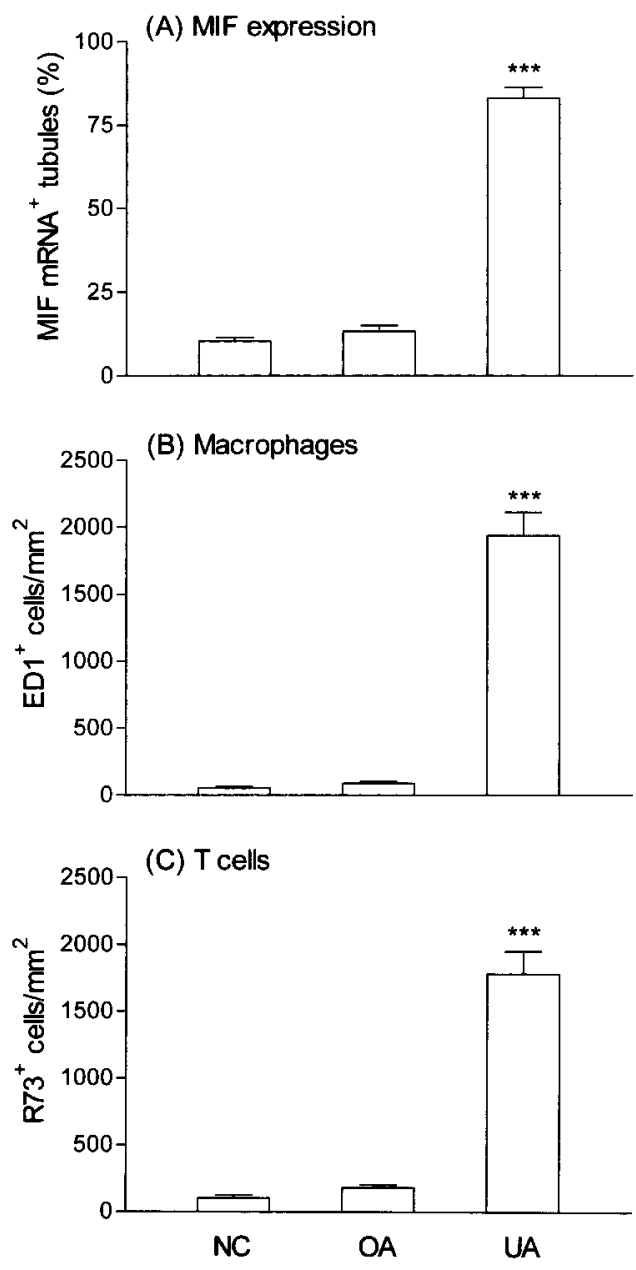

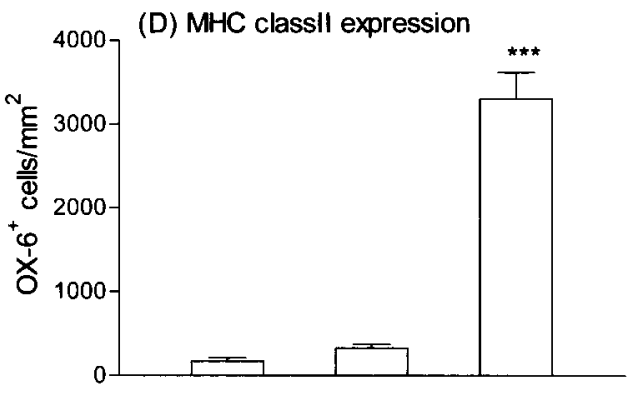

(E) IL-2R expression

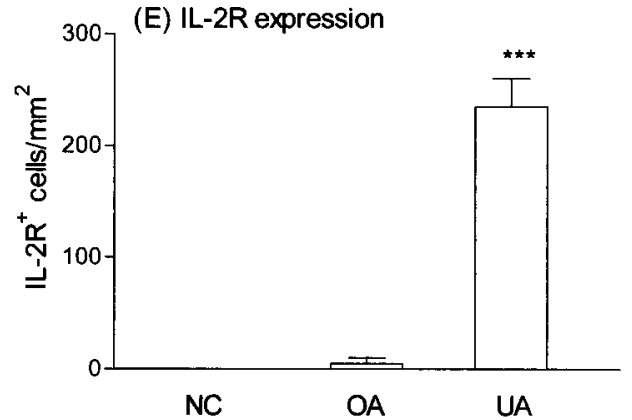

Fig. 5. Quantitation of renal mRNA expression, macrophage, and $T$ cell infiltration, and activation in UA group. Staining of tissue sections for: (A) macrophage migration inhibitory factor (MIF) mRNA, (B) macrophages (EDI $\left.{ }^{+}\right),(\mathrm{C}) \mathrm{T}$ cells $\left(\mathrm{R}^{+} 3^{+}\right),(\mathrm{D})$ $\mathrm{MCH}$ class II $\left(\mathrm{OX}-6^{+}\right)$, and (E) IL-2R $\left(\mathrm{NDS}-6 \mathrm{I}^{+}\right)$were quantitated as described in "Materials and Methods." Data are expressed as the mean \pm SEM. ${ }^{* * *} p<0.001$, compared with oxonic acid (OA) and normal diet control (NC) group. UA, oxonic acid plus uric acid. and $\mathrm{T}$ cell $(\mathrm{r}=0.996, p<0.001)$ accumulation. Further evidence for immune cell activation was shown by the marked expression of MHC class II $\left(\mathrm{OX}-6^{+}\right)$and $\mathrm{IL}-2 \mathrm{R}^{+}$cells by infiltrating macrophages and $\mathrm{T}$ cells within the lesions, compared with normal and OA animals (Fig. 5D, 5E and 6C-6F).

\section{Prominent MIF Expression and Cell Accumulation in the Granulomatous Lesion}

The up-regulation of MIF mRNA expression, and associated increase in macrophage and $\mathrm{T}$ cell infiltration and activation was prominent particularly in the granulomatous lesions, compared with the areas without lesions in UA group (Figs. 3E, 3F and 6B). Indeed, the early deposition of urate crystals within the tubules caused marked tubular epithelial cell proliferation and MIF expression, which resulted in marked macrophage accumulation (Fig. 3E). Cytoplasmic staining for MIF protein in the granulomatous lesions was reduced relatively, compared with MIF mRNA expression. However, multinucleated giant cells found in the granuloma showed strong MIF protein expression (Fig. 3F). Most of infiltrating leukocytes within the granuloma were $\mathrm{EDI}^{+}$macrophages, $\mathrm{R}^{+}{ }^{+} \mathrm{T}$ cells, and to the lesser extent of $\mathrm{W} 3 / 13^{+} \mathrm{R}^{-} 3^{-}$neutrophils (Fig. 7B). Quantitative analysis showed that there was 6-fold increase in tubular MIF mRNA expression (Fig. 7A) and 15 to 20-fold increase in ED ${ }^{+}$macrophages, $\mathrm{W} 3 / 13^{+} \mathrm{T}$ cells, neutrophils, and $\mathrm{R} 3^{+} \mathrm{T}$ cells within the granulomatous lesions, compared with the areas without lesions (Fig. 7). Furthermore, the infiltrating macrophages and $\mathrm{T}$ cells within the granuloma were activated with high expression of MHC class II and IL-2R (Figs. 5D, 5E and 6D, 6F).

\section{Discussion}

It has been known for many years that patients with longstanding gout may develop severe tubulointerstitial disease in which intrarenal 

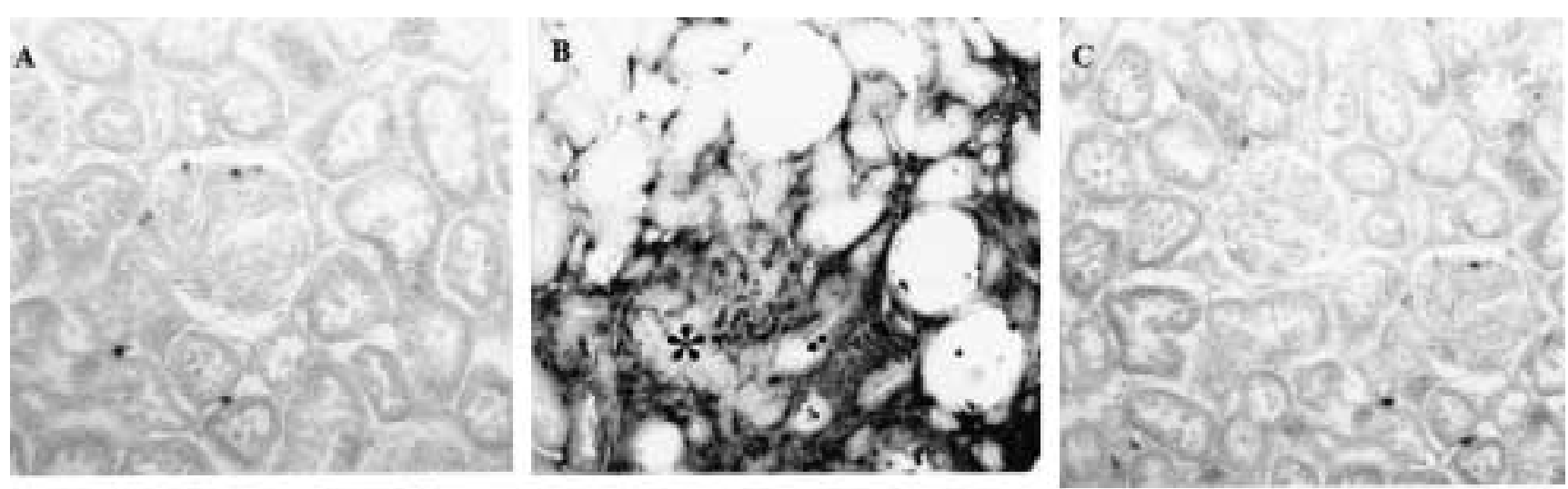
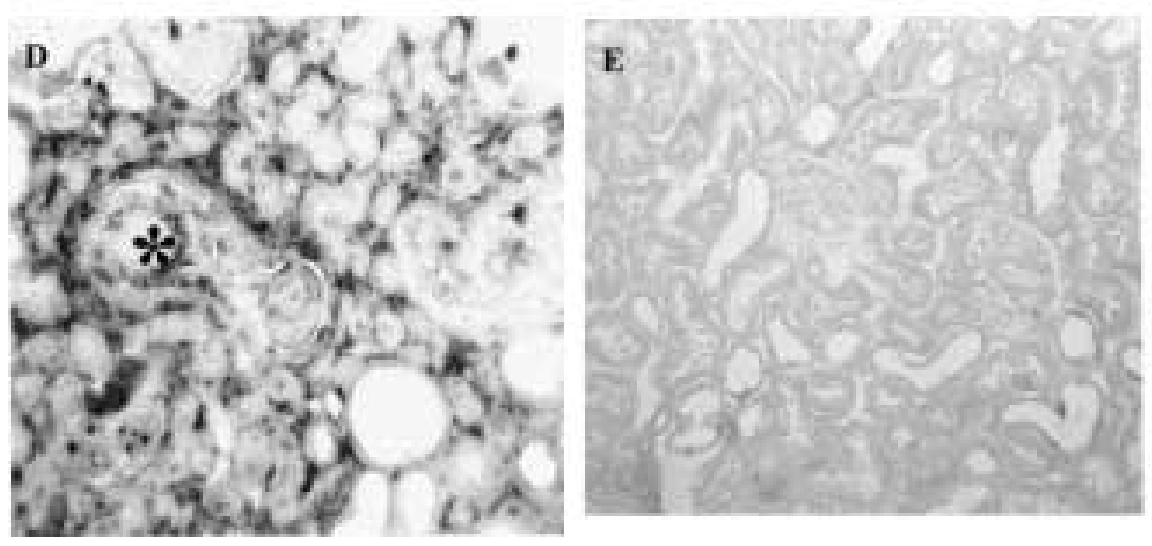

Fig. 6. Immunohistochemistry demonstrating $T$ cell infiltration and immune activation in oxonic acid (OA) group (A, C, E) and oxonic acid plus uric acid (UA) group (B, D, F). (A) Kidney of OA group showing some $\mathrm{R}^{+} 3^{+} \mathrm{T}$ cells within the kidney tissue. (B) Kidney of UA group showing numerous $\mathrm{R}^{+} 3^{+} \mathrm{T}$ cells within granulomatous lesions $(*)$. (C) Kidney of OA group showing few major histocompatibility complex (MHC) class II expressing $\left(\mathrm{OX}-6^{+}\right)$ cells within the tissue. (D) Kidney of UA

medullary urate crystal deposition is prominent. The sites of crystal deposition are often surrounded by a granulomatous inflammatory reaction consistent with a DTH-like reaction. We posited that this reaction might be induced by stimulation of MIF. MIF is recognized to be one of the most important cytokines of the DTH reaction and acts by retarding macrophage migration from the lesion and by activating $\mathrm{T}$ cell responses. Although MIF was originally identified in activated T cells, it is now recognized to be produced by a wide variety of cell types $(14,16)$. Within normal kidney there is some constitutive MIF expression in visceral and parietal epithelial cells, as well as in the renal tubules (16). Recently, it was shown that upregulation of MIF by tubules may play an important role in stimulating local macrophage and group showing strong MHC class II expression $\left(\mathrm{OX}-6^{+}\right)$by most cells within granulomatous lesions. (E) Kidney of OA group showing undetectable interleukin-2R (IL-2R) expression (NDS-61). (F) Kidney of UA group showing strong expression of IL-2R by many infiltrated cells in the injured tubulointerstitial area. Note that some $\mathrm{IL}-2 \mathrm{R}^{+}$cells (arrows) are present within the damaged tubules. Magnifications: 250X (A-C), 100X (D-F).

$\mathrm{T}$ cell accumulation in various renal diseases (16-25).

To investigate the possibility of MIF in UAinduced nephropathy, we induced a model of acute urate nephropathy in rats by administration of oxonic acid (to block endogenous uricase), together with uric acid supplements. Rats with acute urate nephropathy developed significant intrarenal urate crystal deposition in association with tubular injury and renal failure. Tissue obtained from these rats at day 35 documented tubulointerstitial fibrosis and intrarenal urate crystal deposition. Most of the crystals appeared to be intratubular and to be surrounded by a marked inflammatory reaction. A marked increase in MIF mRNA was expressed by the injured tubules and was most marked in the granulomatous lesions. Two-color 


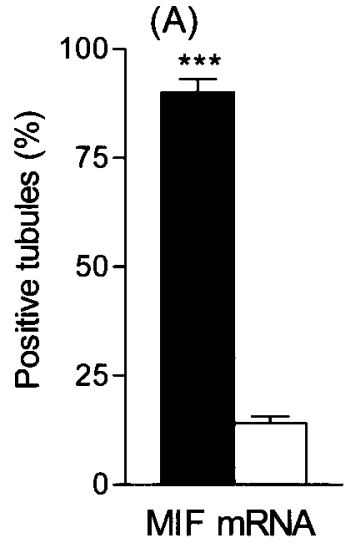

Fig. 7. Quantitation of renal macrophage migration inhibitor factor (MIF) expression, macrophage and $T$ cell infiltration, and activation in granulomatous lesions. Staining of tissue sections for MIF mRNA, T cells and polymorphic neutrophils (PMNs; W3/13 $3^{+}$), T cells $\left(\mathrm{R}^{2} 3^{+}\right)$, macrophages $\left(\mathrm{EDI}^{+}\right)$, major histocompatibility com-

immunostaining confirmed that these lesions were surrounded by giant cells, macrophages, and $\mathrm{T}$ cells, and strongly suggested a DTH-like reaction. Additional evidence was the presence of macrophages and $\mathrm{T}$ cells expressing class II antigens and IL2 receptors, demonstrating that they were immune activated.

One consistent observation was that more cells stained positive for MIF mRNA by in situ hybridization, compared with immunohistochemistry staining of the protein product as previously described in rat kidneys (16). There are two factors that may contribute to this apparent discrepancy. First, there may be differences in the sensitivity of detection of MIF mRNA and protein, even though both use optimized staining methods, based on microwave treatment of paraffin sections. Second, antibody-based immunodetection of MIF protein may not be observed in cells producing MIF, as MIF is stored in the cytoplasm and is released rapidly upon cell activation. Indeed, cellular MIF is often depleted before transcriptional activation can replenish cellular pools (13). Consistent with this possibility was the observation that the sites of UA crystal deposition were associated with a marked up-regulation of MIF mRNA, but very little MIF protein, and by the documentation of a marked increase in urinary MIF protein in UA animals, compared with normal and OA control animals. The source of the MIF likely includes activated tubular epithelial cells and macrophages, and to a lesser extent, $\mathrm{T}$ cells.
(B)

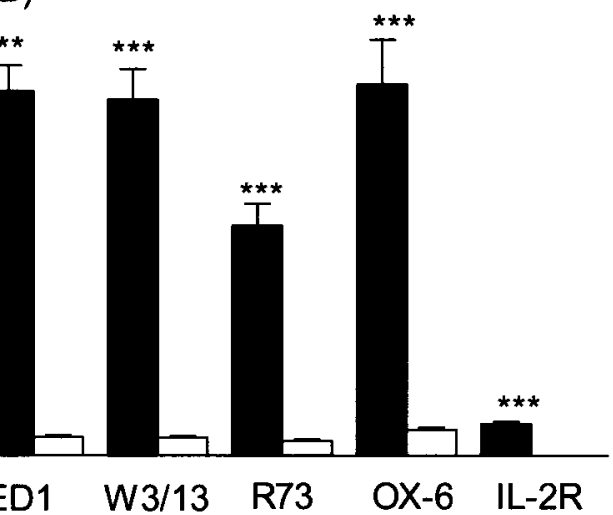

plex $(\mathrm{MCH})$ class II $\left(\mathrm{OX}-6^{+}\right)$, and interleukin-2R (IL-2R; NDS-6 $\mathrm{I}^{+}$) was quantitated in granulomatous lesions (solid bars) and non-granulomatous lesions (opened bars) as described in "Materials and Methods." Data are expressed as the mean \pm SEM. *** $p<$ 0.001 , compared with non-granulomatous tissues.

All have been demonstrated to be a major source of MIF during progressive renal injury in both experimental and human renal disease, including allograft rejection (16-25). The rapid release of MIF may both initiate and amplify the local DTH-like response, ultimately resulting in the macrophage-rich granulomatous lesion. This also may explain why weak MIF mRNA and protein are expressed in uninjured areas in the kidney in association with absent or minimal macrophage accumulation.

These results are consistent with the known ability of MIF to inhibit macrophage migration at sites of inflammation, causing macrophage accumulation at sites of inflammation and inducing macrophage activation (9-11,16-25,36). Indeed, it is these properties that make MIF a pivotal molecule in the DTH response and in experimental crescentic glomerulonephritis. In addition, MIF can stimulate antigenic $T$ cell activation (37), suggesting that local MIF production may enhance antigen recognition.

An interesting finding was that rats fed only oxonic acid produced mild hyperuricemia and hyperuricosuria, but there was no evidence of uric acid crystal deposition. MIF was not up-regulated, compared with normal kidneys. This suggests that up-regulation of MIF by the tubule may require the actual presence of intrarenal urate crystals.

In summary, this study demonstrates that MIF is up-regulated with tubulointerstitial disease induced by uric acid. Up-regulation of 
MIF may cause macrophage and T cell accumulation and activation, leading to progressive tubulointerstitial injury and granulomatous lesion formation. These data suggest that uric acid crystals may initiate a DTH-like reaction via stimulating renal MIF production in the kidney.

\section{Acknowledgements}

This study was supported by U.S. Public Health Service Grants (DK-43422, DK-52121, and DK47659), Hong Kong Research Grants Council (HKU7325/99 M) and a grant from Merck. Y-G. Kim was supported by an International Society of Nephrology Fellowship award.

\section{References}

1. Kjellstrand CM, Cambell DC, von Hartitzsch B, Buselmeier TJ. (1974) Hyperuricemic acute renal failure. Arch. Intern. Med. 133: 349-59.

2. Cameron JS, Moro F, Simmonds HA. (1998) Uric acid and the kidney. In: Davison AM, Cameron JS, Grünfeld J-P, Kerr DS, Ritz E, Winerals CG (eds.). The Oxford Textbook of Clinical Nephrology. Oxford Medical, Oxford, pp. 1157-1173.

3. Emmerson BT, Row PG. Editorial: (1975) An evaluation of the pathogenesis of gouty kidney. Kidney Int. 8: 65-71.

4. Emmerson BT, Thompson L. (1973) The spectrum of hypoxanthine-guanine phosphoribosyltransferase deficiency. Q. J. Med. 42: 423-440.

5. Simmonds HA, Warren DJ, Cameron JS, Potter CF, Farebrother DA. (1980) Familial gout and renal failure in young women. Clin. Nephrol. 14: 176-182.

6. Talbott JH, Terplan KL. (1960) The kidney in gout. Medicine 39: 405-467.

7. Gonick HC, Rubini MD, Gleason IO, Sommers SC. (1965) The renal lesion in gout. Ann. Intern. Med. 62: 667-674.

8. Oliva H, Barat A. (1982) Histopathology of the kidney in percutaneous biopsy in gout. In: Yü T, Berger L (eds.). The Kidney in Gout and Hyperuricemia. Futura Publishing, New York, pp. 175194.

9. Bloom BR, Bennett B. (1966) Mechanism of a reaction in vitro associated with delayed-type hypersensitivity. Science 153: 80-82.

10. David JR. (1966) Delayed hypersensitivity in vitro: its mediation by cell-free substances formed by lymphoid cell-antigen interaction. Proc. Natl. Acad. Sci. U.S.A. 56: 72-77.

11. Bernhagen J, Bacher M, Calandra T, et al. (1996) An essential role for macrophage migration in- hibitory factor in the tuberculin delayed-type hypersensitivity reaction. J. Exp. Med. 183: 277-282.

12. Mikulowska A, Metz CN, Bucala R, Holmdahl R. (1997) Macrophage migration inhibitory factor is involved in the pathogenesis of collagen type II-induced arthritis in mice. J. Immunol. 158: 5514-5517.

13. Bernhagen J, Calandra T, Mitchell RA, et al. (1993) MIF is a pituitary-derived cytokine that potentiates lethal endotoxaemia. Nature 365: 756-759.

14. Bacher M, Meinhardt A, Lan HY, et al. (1997) Migration inhibitory factor expression in experimentally induced endotoxemia. Am. J. Pathol. 150: $235-246$.

15. Calandra T, Bernhagen J, Metz CN, et al. (1995) MIF as a glucocorticoid-induced modulator of cytokine production. Nature 377: 68-71.

16. Lan HY, Mu W, Yang N, et al. (1996) De novo renal expression of macrophage migration inhibitory factor during the development of rat crescentic glomerulonephritis. Am. J. Pathol. 149: 1119-1127.

17. Tesch GH, Nikolic-Paterson DJ, Metz C, et al. (1998) Rat mesangial cells express macrophage migration inhibitory factor in vitro and in vivo. J. Am. Soci. Nephrol. 9: 417-424.

18. Song Q, Nikolic-Paterson DJ, Bacher M, Bucala R, Atkins RC, Lan HY. (1996) Delayed-type hypersensitivity mediates Bowmans capsule rupture in Tamm-Horsfall protein-induced tubulointerstitial nephritis in the rat. Nephrology 2: 417-427.

19. Lan HY, Yang N, Metz C, et al. (1997) TNF- $\alpha$ upregulates renal MIF expression in rat crescentic glomerulonephritis. Mol. Med. 3: 136-144.

20. Lan HY, Bacher M, Yang N, et al. (1997) The pathogenic role of macrophage migration inhibitory factor in immunologically induced kidney disease in the rat. J. Exp. Med. 185: 1455-1465.

21. Yang N, Nikolic-Paterson DJ, Ng YY, et al. (1998) Reversal of rat crescentic glomerulonephritis by blocking of macrophage migration inhibitory factor (MIF): Potential role of MIF in regulating glucocorticoid production. Mol. Med. 4: 413-424.

22. Lan HY, Yang N, Nikolic-Paterson DJ, et al. (2000) Expression of macrophage migration inhibitory factor in human glomerulonephritis. Kidney Int. 57: 499-509.

23. Lan HY, Yang N, Brown FG, et al. (1998) Macrophage migration inhibitory factor expression in human renal allograft rejection. Transplantation 66: 1465-1471.

24. Brown FG, Nikolic-Paterson DJ, Metz C, Bucala R, Atkins RC, Lan HY. (1999) Up-regulation of MIF expression in acute renal allograft rejection in the rat. Clin. Exp. Immunol. 118: 329-336. 
25. Lan HY, Yang N, Nikolic-Paterson DJ, et al. (2000) Expression of macrophage migration inhibitory factor in human glomerulonephritis. Kidney Int. 57: 499-509.

26. Johnson WJ, Stavric B, Chartrand A. (1969) Uricase inhibition in the rat by s-triazines. An animal model for hyperuricemia and hyperuricosuria. Proc. Soc. Exp. Biol. Med. 131: 8-12.

27. Caraway WT. (1963) Uric acid. Stand. Methods Clin. Chem. 4: 239-247.

28. Galantha ED. (1935) Technic for preservation and microscopic demonstration of nodules in gout. Am. J. Clin. Pathol. 5: 165-166.

29. Lan HY, Nikolic-Paterson DJ, Mu W, Atkins RC. (1995) Local macrophage proliferation in the progression of glomerular and tubulointerstitial injury in rat anti-GBM glomerulonephritis. Kidney Int. 48: 753-760.

30. Dijkstra CD, Dopp EA, Joling P, Kraal G. (1985) The heterogeneity of mononuclear phagocytes in lymphoid organs: distinct macrophage subpopulations in the rat recognized by monoclonal antibodies ED1, ED2 and ED3. Immunology 54: 589-599.

31. Hunig T, Wallny HJ, Hartley JK, Lawetzky A, Tiefenthaler G. (1989) A monoclonal antibody to a constant determinant of the rat $T$ cell antigen receptor that induces $\mathrm{T}$ cell activation. Differential reactivity with subsets of immature and mature $\mathrm{T}$ lymphocytes. J. Exp. Med. 169: 73-86.
32. Tellides G, Dallman MJ, Morris PJ. (1989) Mechanism of action of interleukin-2 receptor (IL-2R) monoclonal antibody (MAb) therapy: target cell depletion or inhibition of function? Transplant. Proc. 21: 997-998.

33. Bukovsky A, Presl J, Zidovsky J, Mancal P. (1983) The localization of Thy-1.1, MRC OX 2 and Ia antigens in the rat ovary and fallopian tube. Immunology 48: 587-596.

34. White RAH, Mason DW, William AF, Galfre G, Milstein C. (1978) T lymphocyte heterogeneity in the rat: separation of functional subpopulations using a monoclonal antibody. J. Exp. Med. 148Z: 664-673.

35. Lan HY, Mu W, Nikolic-Paterson DJ, Atkins RC. (1995) A novel, simple, reliable, and sensitive method for multiple immunoenzyme staining: use of microwave oven heating to block antibody crossreactivity and retrieve antigens. J. Histochem. Cytochem. 43: 97-102.

36. Calandra T, Bernhagen J, Mitchell RA, Bucala R. (1994) The macrophage is an important and previously unrecognized source of macrophage migration inhibitory factor. J. Exp. Med. 179: 1895-1902.

37. Bacher M, Metz CN, Calandra T, et al. (1996) An essential regulatory role for macrophage migration inhibitory factor in T-cell activation. Proc. Natl. Acad. Sci. U.S.A. 93: 7849-7854. 